\title{
Two new species of the genus Geranomyia Haliday, 1833 (Diptera: Limoniidae) from Japan, with notes on the Japanese species
}

\author{
Ава новых вида рода Geranomyia Haliday, 1833 из Японии с \\ замечаниями по распространению и таксономии японских \\ комаров болотниц (Diptera: Limoniidae)
}

\author{
D. Kato \\ A. Като \\ Echigo-Matsunoyama Museum of Natural Sicences, «Kyororo», 1712-2 Matsunoyama, Tôkamachi 942-1411 Japan. E-mail: \\ hehemanpuldoa.d4@gmail.com. \\ Музей Естественных наук Ичиго-Мацунояма, «Кьороро», 1712-2 Мацунояма, Токамати 942-1411 Япония.
}

Key words: Geranomyia, Japan, Limoniidae, new species, synonym, taxonomy.

Ключевые слова: Geranomyia, Япония, Limoniidae, новый вид, синоним, таксономия.

Abstract. The Japanese species of Geranomyia are revised. Two new species, Geranomyia iriomotensis Kato, sp.n. and G. paucipuncta Kato, sp.n., are described, and G. snyderi chichiensis Alexander, 1972 is synonymized with G. snyderi snyderi Alexander, 1972. Images of the external appearance, wings, and male terminalia, and distributions of the Japanese species are shown. A key to the Japanese species is provided.

Резюме. В работе ревизованы виды рода Geranomyia Японии. Описаны два новых для науки вида: G. iriomotensis Kato, sp.n. и G. paucipuncta Kato, sp.n., Geranomyia snyderi chichiensis Alexander, 1972 синонимизорован с номинативным подвидом G. snyderi snyderi Alexander, 1972. Приведены изображения внешнего вида, крыльев, гениталий самцов и даны карта распространения и определительная таблица видов, обитающих в Японии.

\section{Introduction}

Geranomyia Haliday, 1833 is a genus of the subfamily Limoniinae and is easily distinguished from the other genera of the subfamily by the considerably elongate labellum. The genus includes 342 species ( 30 species in the Palaearctic, 16 species in the Nearctic, 160 species in the Neotropical, 32 species in the Afrotropical, 75 species in the Oriental, and 39 species in the Australasian / Oceanian Regions) [Oosterbroek, 2020]. In the past several years, new species of the genus have been described from Korea and Kunashir [Podenas, 2016], China [Zhang et al., 2016; Qian, Zhang, 2020], and the Dominican Republic [Gavryushin, 2018].

In Japan, 13 species including two subspecies have been recorded: G. argentifera de Meijere, 1911; G. avocetta Alexander, 1913; G. feuerborni (Alexander, 1931); $G$. gifuensis Alexander, 1921; G. gravelyana (Alexander, 1942); G. hakoneana (Alexander, 1955); G. multipuncta Alexander, 1922; G. pictorum (Alexander, 1929); G. radialis (Alexander, 1930a); G. sakaguchii Alexander, 1924;
G. semjina Podenas, 2016; G. snyderi snyderi (Alexander, 1972); G. snyderi chichiensis (Alexander, 1972); G. sparsiguttata (Alexander, 1937) [Nakamura, 2014; Kato, 2020]. In this study, Japanese species of the genus are revised, including two new species and a synonym of subspecies. A key to the Japanese species, images of the habitus and wings, drawings of the male terminalia, and distributions of the species are provided.

\section{Materials and methods}

Samples were mainly collected by insect nets and light traps (LT), and preserved in the Biosystematic Laboratory, Kyushu University, Japan (BLKU). Specimens including types in the collection of the National Museum of Natural History, Smithsonian Institution, Washington D.C., USA (USNM) were examined. Information of collector and depository of specimens in 'Material' is omitted in case it is by the author and preserved in BLKU. General descriptions were based on examining dried specimens. For drawings and descriptions of male terminalia, they were heated in a solution of $10 \% \mathrm{KOH}$ for several minutes and then rinsed in $70 \%$ ethanol with 3\% acetic acid for neutralization, and observed in pure glycerin. The treated terminalia were preserved in genitalia tubes filled with glycerin and the tubes were pinned below the body remains. Terminology mainly follows Cumming and Wood [2017] for general description and Ribeiro [2006] for gonostyli of male terminalia.

\section{Taxonomy \\ Geranomyia iriomotensis Kato, sp.n. Figs $1-7,68$.}

Diagnosis. Brownish species with sparsely patterned wing markings (Fig. 1). Head grayish. Prescutum with five weakly dark stripes. Wing with six dark costal spots (Fig. 2). 


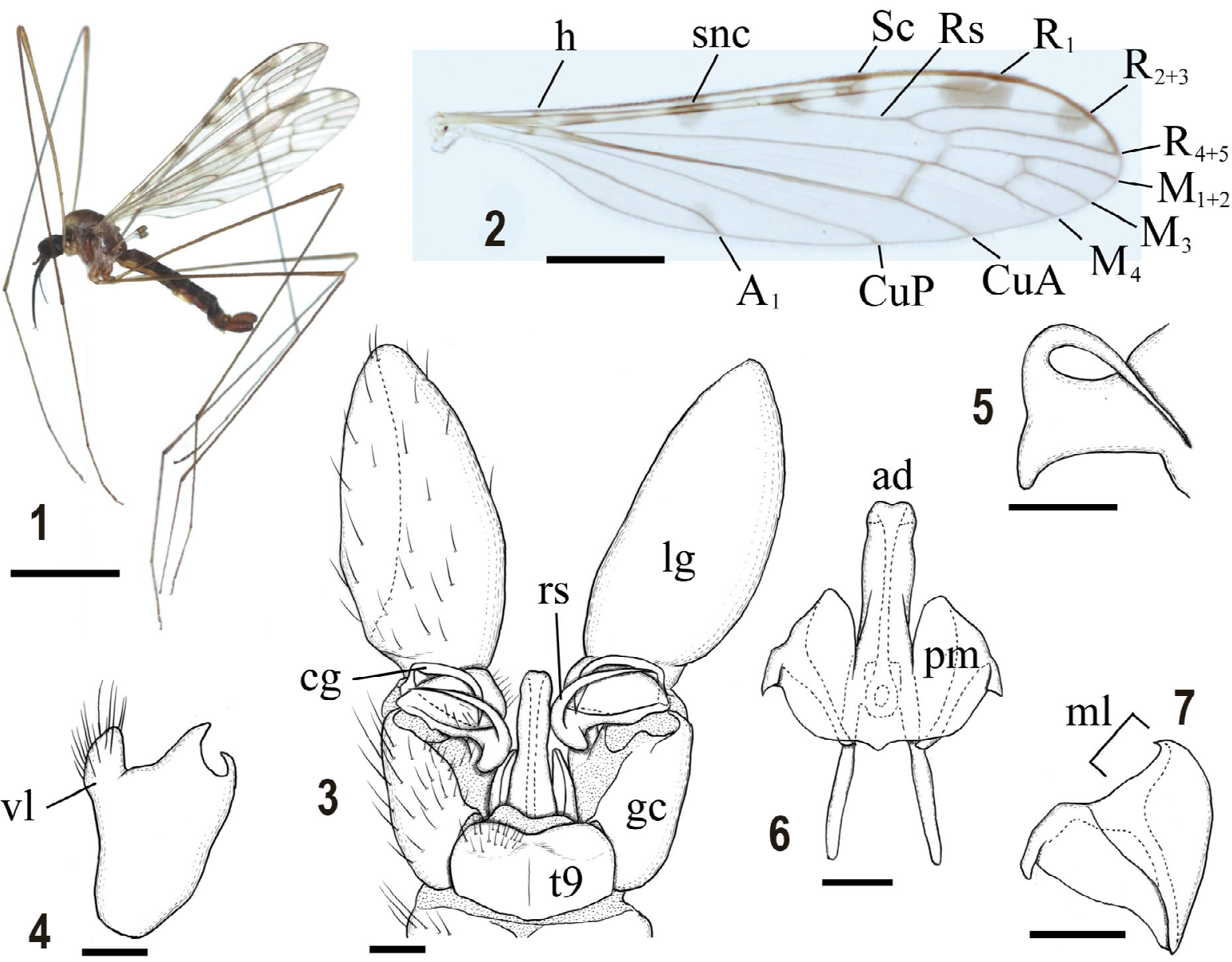

Figs 1-7. Geranomyia iriomotensis sp.n.: 1 - habitus in lateral view; $2-$ wing; 3 - male terminalia in dorsal view; $4-$ gonocoxite in lateroventral view; 5 - rostral lobe of gonostylus, outer surface; 6 - aedeagal complex in dorsal view; 7 - paramere, outer surface. Abbreviations: ad — aedeagus; cg — clasper of gonostylus; gc - gonocoxite; lg — lobe of gonostylus; ml - mesalapical lobe; pm - paramere; rs - rostral spine; snc - supernumerary crossvein; $\mathrm{t} 9$ - tergite 9; vl - ventromesal lobe. Scale bars: $1-3 \mathrm{~mm} ; 2-1 \mathrm{~mm} ; 3-7-0.1 \mathrm{~mm}$.

Рис. 1-7. Geranomyia iriomotensis sp.n.: 1 - внешний вид сбоку; 2 - крымо; 3 - гениталии самца дорсально; $4-$ гонококсит матеровентрально; 5 - передняя часть гоностиля, снаружи; $6-$ части эдеагуса, дорзально; 7 - парамеры, снаружи. Аббревиатуры: ad - эдеагус; $\mathrm{cg}$ - усик гоностиля; gc - гонококсит; lg - доля гоностиля; $\mathrm{ml}$ - средне-вершинная Аоля; pm - парамеры; rs - вершинная спикула; snc - дополнительная поперечная жилка; t9 - тергит 9; vl - нижнесрединная доля. Масштаб: $1-3$ мм; $2-1$ мм; $3-7-0,1$ мм.

Legs mainly yellow to brown, paler on segments proximal to base of femora. Halter dark brown, stem yellowish. Abdomen dark brown on tergites. Male terminalia with rostral lobe bearing one curved stout spine, about as long as clasper of gonostylus (Fig. 5).

Material. Holotype: $\bigcirc^{\top}$, Japan, Nansei Islands, Okinawa, Iriomote Is., Taketomi-cho, Uehara, Urauchi-gawa River, Alt $50 \mathrm{~m}$, 14.III.2016. Paratypes: Japan: [Nansei Islands] Iriomote Is.: $160^{7} O^{x}$, $69+9$, same data as holotype. $60^{7} 0^{2}$, Komi, Mairagawa River, Alt. 20 m, 15.III.2016. 10', Haeminaka, Alt. 170230 m, 19.V.2016. 10, 19, Haeminaka-Haemi, Alt. 220 m, 15.III.2016.

Description. Male. Body length excluding rostrum and labellum: 4.6-5.2 mm. Wing length: 4.9-6.1 mm. Rostrum + labellum: $1.6-1.9 \mathrm{~mm}$. Head dark gray, anterior part of vertex lighter gray, slightly narrower than scape, posterior part of vertex with central gray line in anterodorsal view; rostrum and palpus dark brown; antenna about $2 / 3$ length of rostrum, dark brown, flagellomeres bacilliform.
Thorax with pronotum brownish gray to dark brown, caudal margin of antepronotum and lateral side of postpronotum usually whitish; prescutum ocherous to brown, yellowish or grayish on lateral side, with five weakly darker stripes in dorsal and posterodorsal views, middle one on anterior part, lateral ones on posterior part, outer one shortest; scutum ocherous to brown, scutal lobe with two dark stripes, anterior one oblique, posterior one longitudinally straight, these two stripes often fused with each other, forming large dark area on anterior $2 / 3$, interspace between scutal lobes ocherous to grayish ocherous; scutellum yellowish ocherous to grayish ocherous, sometimes darkened at middle of base; postnotum yellowish ocherous to brown, laterotergite sometimes slightly paler; pleuron pale whitish yellow to ocherous, slightly darker on anepisternum and anepimeron. Wing (Fig. 2) tinged with brown, bearing six dark costal spots, first one at supernumerary crossvein in cell sc, second one at origin of Rs, third one at crossvein sc-r, fourth one as stigma, 
oval and largest, fifth and sixth ones at tips of $\mathrm{R}_{2+3}$ and $\mathrm{R}_{4+5}$ respectively, sometimes indistinctly dark spot present near MA; tip of $\mathrm{A}_{1}$ often with small dark spot; cell bm sometimes with one to three tiny spots; cord and outer end of cell $\mathrm{d}$ sometimes seamed with dark brown; crossvein $\mathrm{r}-\mathrm{r}$ slightly proximal to or at middle of $\mathrm{R}_{2+3}$; Rs curved at base; crossvein $\mathrm{m}$-cu situated less than half length of itself from inner end of cell d. Legs with coxae and trochanters pale whitish yellow to ocherous; succeeding segments of legs dusky yellow to brown, bases of femora narrowly yellowish. Halter dark brown, stem yellowish entirely or basally.

Abdomen dark brown, pale whitish yellow to ocherous on sternites. Male terminalia (Figs 3-7) ocherous to brown, with tergite 9 shallowly and weakly concaved at middle of posterior margin; gonocoxite with ventromesal lobe about $1 / 3$ length of gonocoxite; clasper of gonostylus sickle-shaped, strongly curved especially at basal $1 / 4$; lobe of gonostylus bacilliform, 1.5-2 times as long as gonocoxite, rostral lobe with one curved stout spine, about as long as clasper of gonostylus; paramere with mesal-apical lobe gradually narrowed to tip, extreme tip acute and directed dorsally; aedeagus straight rod-shaped in dorsal view, tip bilobed, narrow and bent posteroventrally in lateral view.

Female. Body length excluding rostrum and labellum: 4.0-6.2 $\mathrm{mm}$. Wing length: $4.5-5.5 \mathrm{~mm}$. Rostrum + labellum: $1.5-1.9 \mathrm{~mm}$. Resembling male except terminalia. Ovipositor with tergites brown to dark brown, distal $1 / 4$ of tergite 10 yellowish; sternite 8 pale dusky yellow, sometimes brownish; cercus and hypogynial valve amber-color, anterior corner of hypogynial valve dark brown; cercus about as long as tergite 10 , straight and thin, obtuse at tip; hypogynial valve about 1.5 times as long as sternite 8 , tip ending at level of middle of cercus.

Distribution. Japan (Nansei Islands) (Fig. 68).

Etymology. This species is named after the type locality, Iriomote Island. Feminine adjective in nominative singular.

Remarks. This species is similar to G. spectata (Alexander, 1937), which was described with a single female from China, but is differentiated from it by the following characters: prescutum ocherous to brown with five slightly darker stripes (almost uniform plumbeous-gray with two slightly darker stripes in G. spectata); wing (Fig. 2) usually with dark spot near crossvein $\mathrm{h}$ and tip of $\mathrm{A}_{1}$ respectively (without these spots in G. spectata); cell $\mathrm{r}_{2+3}$ without small spot just posterior to stigma (with small weak spot just posterior to stigma in G. spectata); abdomen with tergites monocolorous (vaguely paler on caudal margins of intermediate tergites in G. spectata). The male terminalia of this species is somewhat similar to G. multipuncta, but the rostral spine is as about long as the clasper of gonostylus in this species (Fig. 5) and about twice as long as the one in G. multipuncta (Fig. 46).

\section{Geranomyia paucipuncta Kato, sp.n.} Figs 8-15, 68.

Diagnosis. Brownish species with sparsely patterned wing markings (Fig. 8). Head grayish. Prescutum with three weakly dark stripes. Wing with five small dark costal spots, sometimes indistinct except stigma (Figs. 9-10). Legs mainly dusky yellow to brown, paler on segments proximal to bases of femora. Halter dark brown, stem yellowish. Abdomen dark brown on tergites. Male terminalia with rostral lobe bearing long arched spine, about 1/2 length of clasper of gonostylus (Fig. 13).

Material. Holotype: $\mathrm{O}^{7}$, Japan, Honshu, Aichi, Seto-shi, Iwaya-chô, near Iwayadô Park, Alt. 300 m, 4.V.2016. Paratypes:
Japan: [Honshu] 19 , same data as holotype. $10^{2}$, same data as holotype except 17.VII.2016. 10 $0^{7}$, Okayama, Maniwa-shi, Hiruzen-Kamifukuda, Nawashirodani-gawa River, Alt. 600 m, 1.V.2016. [Shikoku] 10', Ehime, Toon-shi, Kawanouchi, 20.IV.2014. [Kyushu] 10', Fukuoka, Hisayama-machi, Ino, Ino Dam, Alt. 230 m, 1.V.2015. 10', Kagoshima, Kirishima-shi, Hayato-chô-Kareigawa, around Amori-gawa River, Alt. 50 m, 28.IV.2018. [Nansei Islands] Yakushima Is.: $20^{7} 0^{7}$, near Shirataniunsui-kyô Valley, Alt. 600 m, 25.IV.2018. 10", same data as previous except 27.IV.2018. Amamiôshima Is.: 10' Setouchi-chô, Agina, east of Agina-gawa River, Alt. 300 m, 3.IV.2019. $40^{7} \mathrm{O}^{7}, 4$ 오, Yamato-son, Yuwangama, Alt. $250 \mathrm{~m}$, 31.III.2019. Tokunoshima Is.: 1우, Tokunoshima-cho, Kametsu, near trailhead of Mt. Inokawa-dake, Alt. 300 m, 1.IV.2019. 107, Tokunoshima-cho, Todoroki, near Mt. Sasontsuii-dake, Alt. 200 m, 2.IV.2019. Okinawa Is.: 10" , Nago-shi, Genka, Mt. Ubashi-yama, Alt. $20 \mathrm{~m}, 23 . V .2016$. 2090, same data as previous except 12.III.2016, Alt. 100 m. 10", Kunigami-son, Yona, Mt. Fuenchiji-dake, Alt. 250 m, 12.III.2016. 19, same data as previous except 21.V.2016. 10" , same data as previous except 23.V.2016. 1엉 Kunigami-son, Hiji, Ufugi Nature Museum, Alt. $10 \mathrm{~m}$, 10.III.2016.

Description. Male. Body length excluding rostrum: $4.7-$ $7.1 \mathrm{~mm}$. Wing length: $5.2-8.3 \mathrm{~mm}$. Rostrum + labellum: 1.8 $2.6 \mathrm{~mm}$. Head dark gray, anterior part of vertex lighter gray, slightly narrower than scape, posterior part of vertex with central gray line in anterodorsal view; rostrum and palpus dark brown; antenna about 2/3 length of rostrum + labellum, dark brown, flagellomeres bacilliform.

Thorax with pronotum dark brownish gray, postpronotum and caudal margin of antepronotum yellowish; mesonotum generally yellowish ocherous to brownish gray; prescutum lighter yellow or lighter gray on lateral margin, with three indistinctly dark stripes, middle one on anterior half to whole length of prescutum, often disappeared, lateral one on posterior $1 / 2$ to $2 / 3$ of prescutum, posterolateral corner of prescutum sometimes weakly dark; scutal lobe weakly darkened medially, or with two weak stripes, outer one oblique, situated on anterior part, inner one along close to inner margin; scutellum occasionally with weakly dark stripe at middle; pleuron pale yellow to ocherous. Wing (Figs 9-10) tinged with brownish yellow, stigma oval, dark brown; small dark spot at each of supernumerary crossvein in cell sc, crossvein sc-r, origin of Rs, and tip of $\mathrm{R}_{2+3}$, these costal spots except stigma smaller in specimens from Palaearctic region of Japan (Fig. 9) and larger in those from Oriental region of Japan (Fig. 10); weakly dark spot sometimes present at tip of $A_{1}$; crossvein $r-r$ slightly proximal to middle of $R_{2+3}$; Rs curved at base; crossvein m-cu usually proximal to base of cell d, sometimes slightly distal to base of cell d. Legs with coxae and trochanters yellow to brown; succeeding segments of legs dusky yellow to brown, bases of femora sometimes narrowly more yellowish, tips of tibiae sometimes narrowly and weakly darkened, distal two tarsomeres and tips of tarsomeres 1 to 3 sometimes darkened. Halter dark brown, stem ocherous basally.

Abdomen brown to dark brown, dusky yellow to ocherous on sternites. Male terminalia (Figs 11-15) sometimes paler than pregenital tergites; tergite 9 roughly rectangular, widely and shallowly concaved at middle of posterior margin; gonocoxite with ventromesal lobe about $1 / 3$ length of gonocoxite, middle part as wide as basal one; clasper of gonostylus sickle-shaped, strongly curved on distal half; lobe of gonostylus bacilliform, about 2.5 times as long as gonocoxite; rostral lobe with long arched spine, about $1 / 2$ length of clasper of gonostylus, directed laterally to lateroventrally on apical part; paramere with basal part large, mesal-apical lobe 

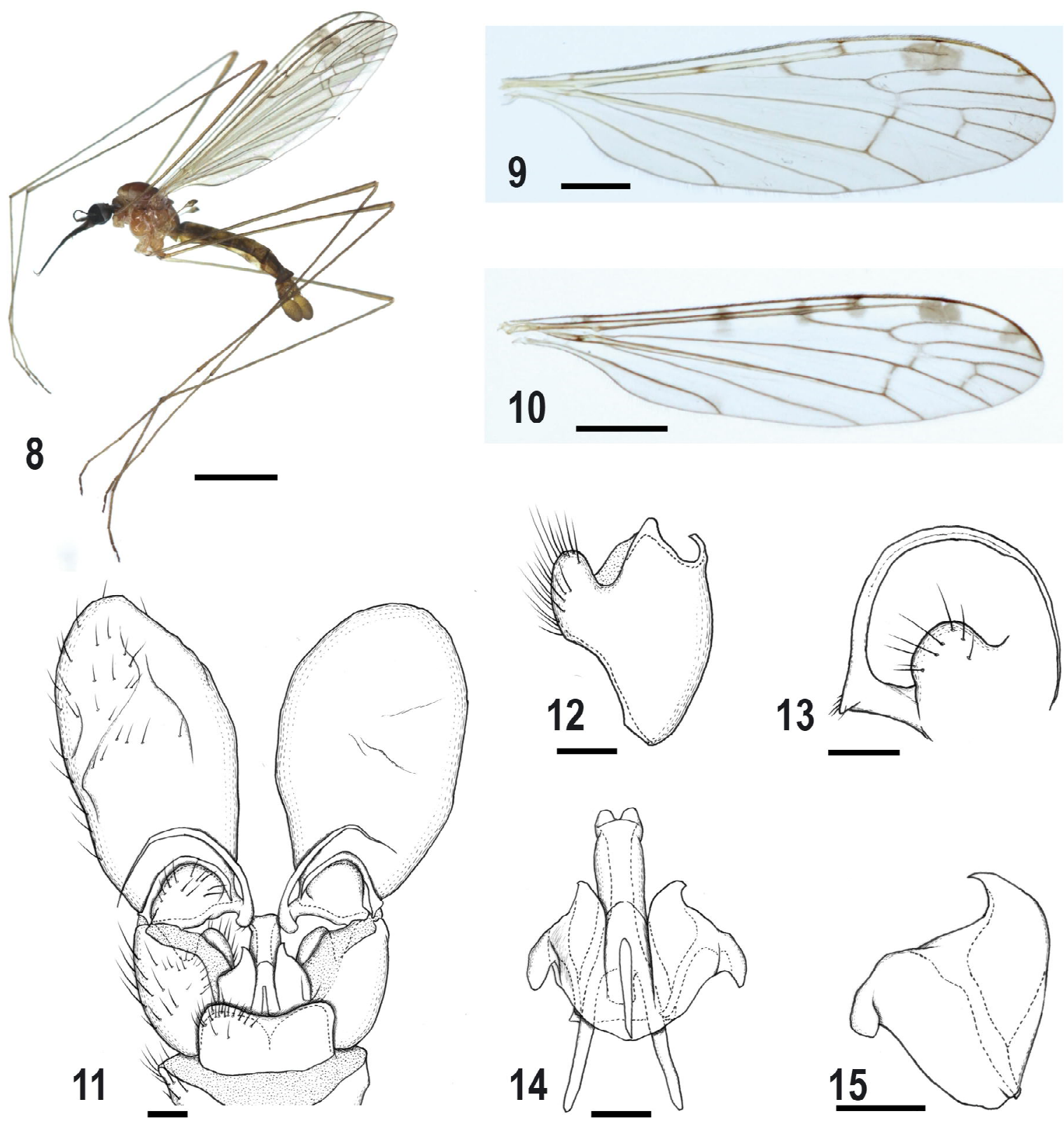

Figs 8-15. Geranomyia paucipuncta sp.n.: 8 - habitus in lateral view; 9-10 - wing; 11 - male terminalia in dorsal view; 12 - gonocoxite in lateroventral view; 13 - rostral lobe of gonostylus, outer surface; 14 - aedeagal complex in dorsal view; 15 - paramere, outer surface. Scale bars: $8-3 \mathrm{~mm}$; 9-10 - $1 \mathrm{~mm} ; 11-15-0.1 \mathrm{~mm}$.

Figs 8-15. Geranomyia paucipuncta sp.n.: 8 - внешний вил сбоку; 9-10 - крымо; 11 - гениталии самца дорсально; $12-$ гонококсит матеровентрально; 13 - передняя часть гоностиля, снаружи; 14 - части эдеагуса, дорсально; 15 - парамеры, снаружи. Масштаб: 8 - 3 мм; 9-10-1 мм; 11-15 - 0,1 мм.

wide claw-shaped, curved dorsally at tip, about $1 / 4$ as long as remainder of paramere; aedeagus straight rod-shaped and slightly narrow on distal $1 / 3$ in dorsal view, curved ventrally on distal $1 / 3$, tip bilobed.

Female. Body length excluding rostrum and labellum: 5.6$6.9 \mathrm{~mm}$. Wing length: $6.1-7.8 \mathrm{~mm}$. Rostrum + labellum: 1.8 $2.5 \mathrm{~mm}$. Resembling male except terminalia. Ovipositor brown, often darker on tergites 8 and 9 , sometimes yellowish on sternite 8 ; cercus and hypogynial valve amber-color, some- times reddish, basal part of hypogynial valve sometimes weakly brownish; cercus about as long as tergite 10 , straight and thin, obtuse at tip; hypogynial valve about 1.5 times as long as sternite 8 , tip ending at level of anterior $1 / 3$ to $1 / 2$ of cercus.

Distribution. Japan (Honshu, Shikoku, Kyushu, and Nansei Islands) (Fig. 68).

Etymology. The specific name paucipuncta is derived from the Latin adjective paucus (few) + punctus (punctured, marked with points), meaning the fewer small spots on the 
wing of this species compared to the morphologically similar species, G. multipuncta. Feminine adjective in nominative singular.

Remarks. This species is similar to G. multipuncta in terms of structure of male terminalia, but is differentiated from it by the following characters: wing with spots on costal region very small or indistinct, cell bm with at most three spots (Figs 9-10) (costal spots large and distinct, cell bm with abundant small spots in G. multipuncta (Fig.22)); male terminalia with base of ventromesal lobe of gonocoxite as wide as middle part (Fig. 12) (distinctly narrower than middle part in G. multipuncta).
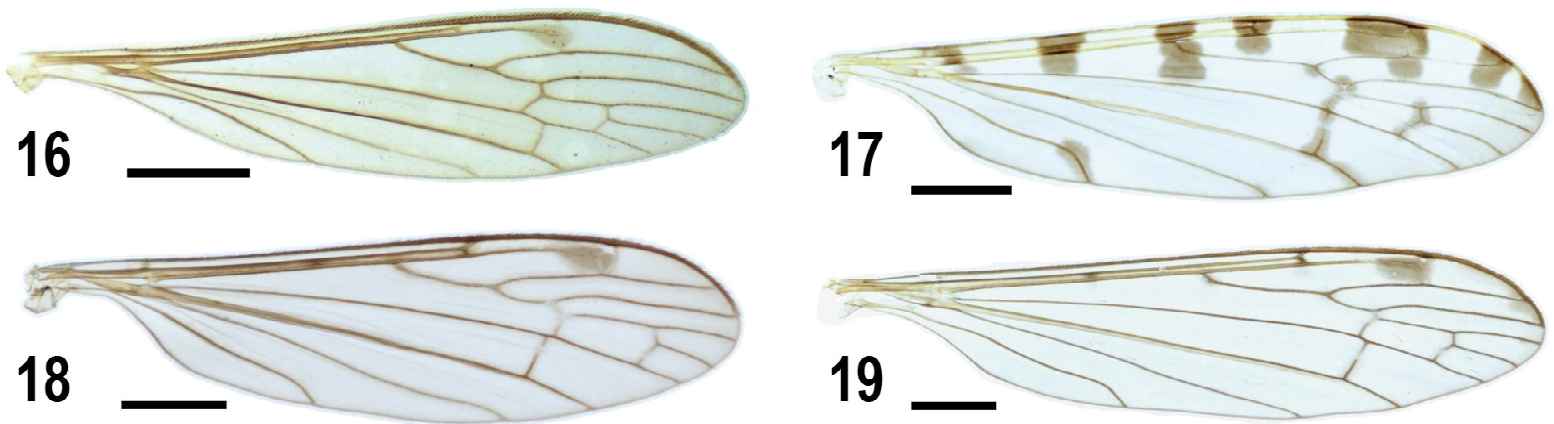

Figs $16,29,41,53,66$

Geranomyia argentifera de Meijere, 1911: 29. Type locality: Indonesia, Java.

Geranomyia argentifera: Kato [2020: 24].

Diagnosis. Blackish species with unpatterned wing except stigma (Fig. 16). Head silvery grey anteriorly. Eyes distinctly dichoptic. Prescutum with two silvery spots at lateral margin. Legs mainly yellow to brown, paler on coxae and trochanters. Halter pale yellow. Abdomen brown to dark brown on tergites. Male terminalia with rostral lobe bearing
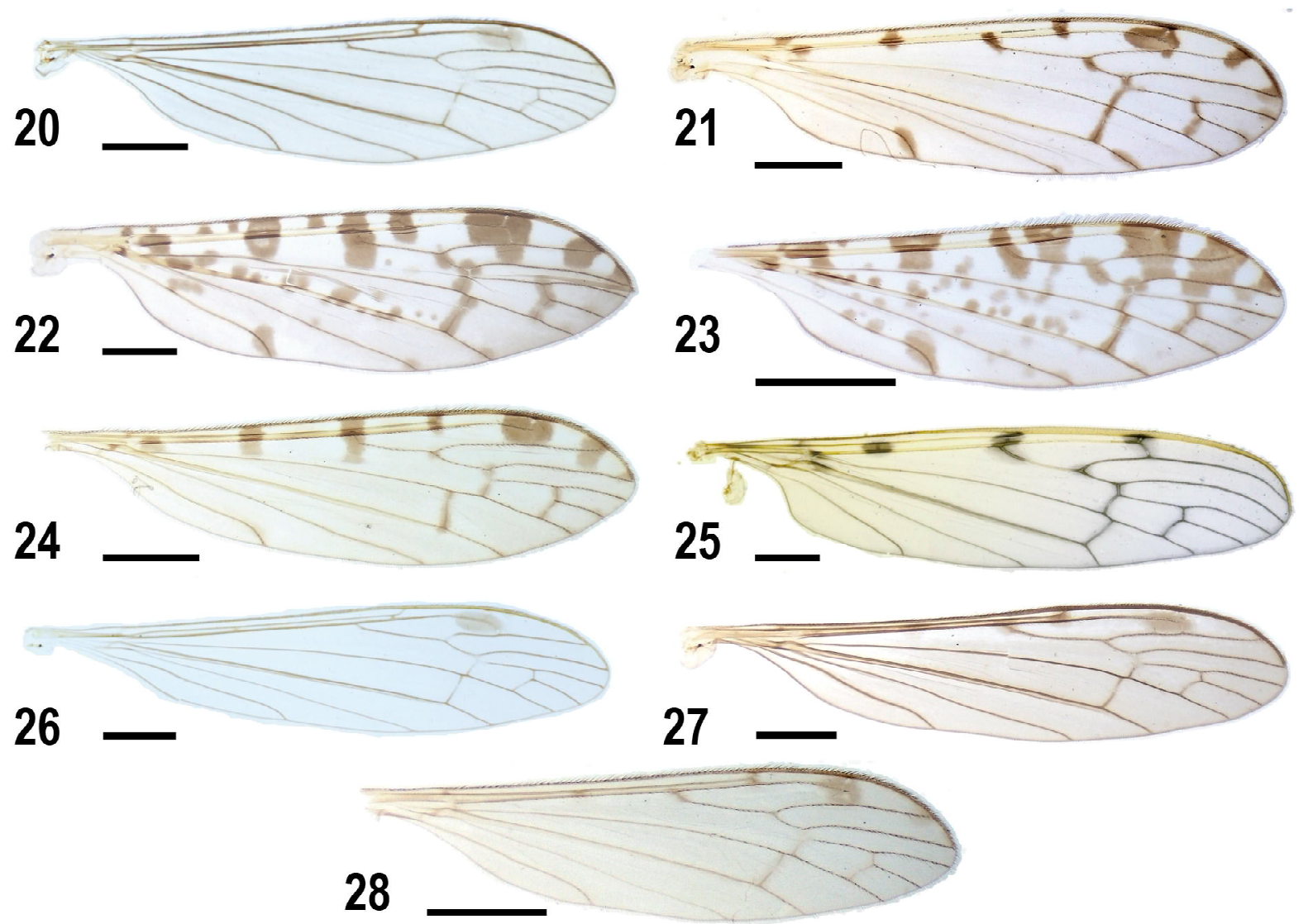

Figs 16-28. Wings of Japanese Geranomyia spp.: 16 - G. argentifera; 17 - G. avocetta; 18 - G. feuerborni; 19 - G. gifuensis; 20 - G. gravelyana; 21 - G. bakoneana; 22 - G. multipuncta; 23 - G. pictorum; $24-$ G. radialis; 25 - G. sakaguchii; $26-$ G. semjina; 27 - G. snyderi; 28 - G. sparsiguttata. Scale bars: $1 \mathrm{~mm}$.

Рис. 16-28. Крымья японских видов рода Geranomyia spp.: 16 - G. argentifera; 17 - G. avocetta; 18 - G. feuerborni; 19 G. gifuensis; 20 - G. gravelyana; 21 - G. hakoneana; 22 - G. multipuncta; 23 - G. pictorum; 24 - G. radialis; 25 - G. sakaguchii; 26 - G. semjina; 27 - G. snyderi; 28 - G. sparsiguttata. Масштаб: 1 мм. 

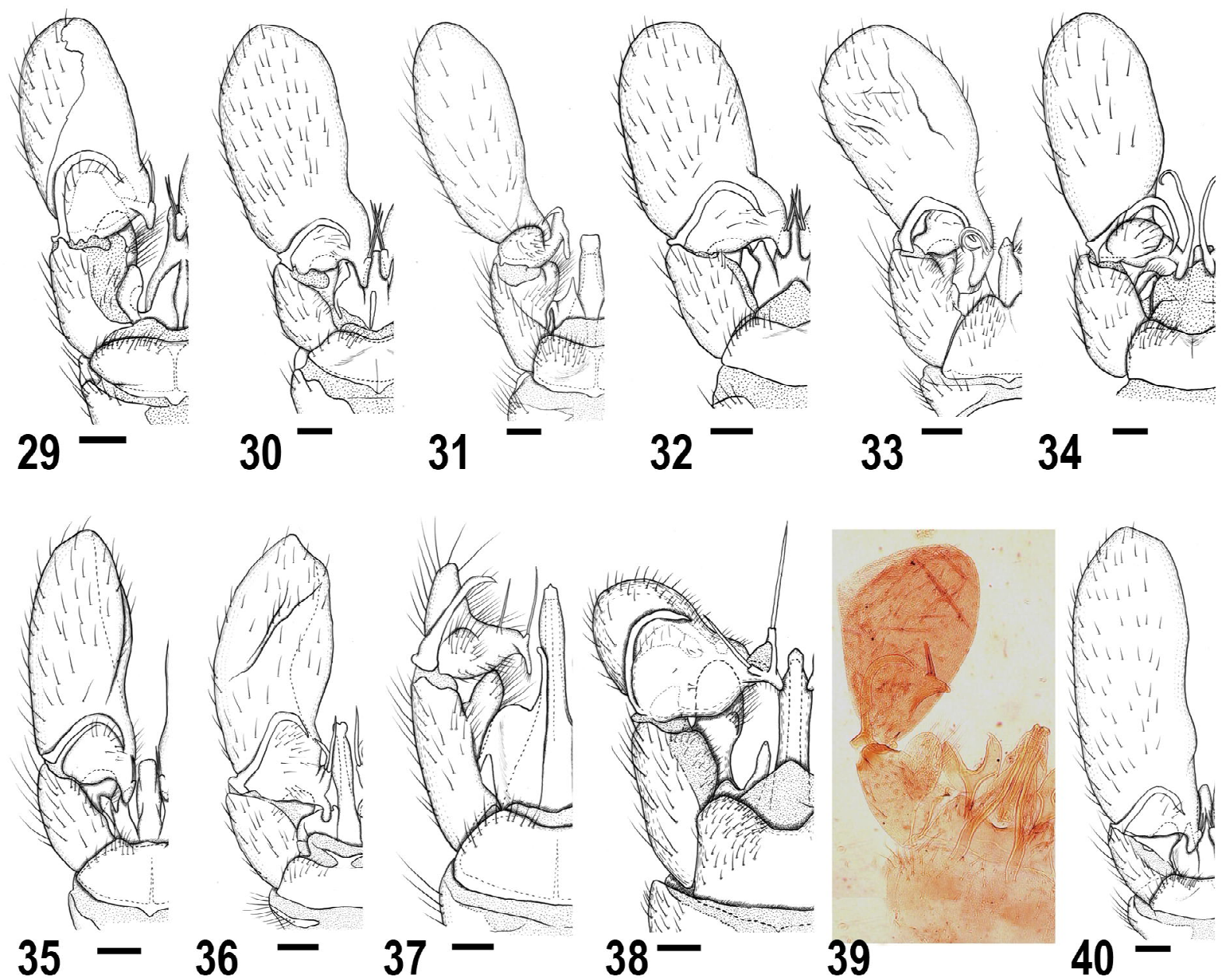

Figs 29-40. Male terminalia of Japanese Geranomyia spp., dorsal view (left parts omitted): 29 - G. argentifera; 30 G. avocetta; 31 - G. feuerborni; 32 - G. gifuensis; 33 - G. gravelyana; 34 - G. multipuncta; 35 - G. pictorum; 36 - G. radialis; 37 - G. sakaguchii; 38 - G. semjina; 39 - G. snyderi; 40 - G. sparsiguttata. Scale bars: $0.1 \mathrm{~mm}$.

Рис. 29-40. Гениталии самџов (дорсально без мевой стороны) японских видов рода Geranomyia spр.: 29 - G. argentifera; 30 - G. avocetta; 31 - G. feuerborni; 32 - G. gifuensis; 33 - G. gravelyana; 34 - G. multipuncta; 35 - G. pictorum; $36-$ G. radialis; 37 - G. sakaguchii; 38 - G. semjina; 39 - G. snyderi; 40 - G. sparsiguttata. Macшта6: 0,1 мм.

two short spines (Fig. 41). Mesal-apical lobe of paramere with row of setae (Fig. 53).

Distribution. Japan (Nansei Islands) (Fig. 66), China, Taiwan, Indonesia, and Philippines.

\section{Geranomyia avocetta Alexander, 1913}

Figs 17, 30, 42, 54, 65.

Geranomyia avocetta Alexander, 1913: 205. Type locality: Japan, Tokyo.

Limonia (Geranomyia) avocetta: Alexander [1953: 162].

Geranomyia avocetta: Nakamura [2002: 170]; Nakamura [2014: 46].

Diagnosis. Greyish species with heavily patterned wing. Prescutum usually with three dark stripes. Wing with large dark costal spots, tip of $\mathrm{A}_{1}$ with dark spot, cord and outer end of cell d seamed with brown (Fig. 17). Legs mainly yellow to brown, paler on segments proximal to bases of femora. Halter brown to dark brown, stem yellowish. Abdomen dark brown on tergites. Male terminalia with rostral lobe bearing two relatively long spines (Fig. 42). Mesal-apical lobe of paramere weakly bilobed at tip (Fig. 54).

Material. Paratype: Japan: [Honshu] 10', Tokio (Tokyo), ?.VIII.1912, Kuwana leg. (USNM). Non-types: Japan: [Hokkaido] $10^{7}$, Iwamizawa-shi, Kurisawa-chô-Nishimanzi, Manzi Spa, Alt. 430 m, 24.VI.2014. [Honshu] 1옹, Aomori, Nishimeyamura, Kawaratai, Ôkawa-rindô Path, Alt. 300 m, 4.X.2014. 1웅 Aomori, Nishimeya-mura, Kawaratai, The Shirakami Natural Science Park, Hirosaki Univ., Alt. 255 m, 25.V.2014. 19, Aomori, Towada-shi, Okuse, Tsutanuma-rindô Path, Alt. 460 m, 8.X.2014. 10", (Tokyo), Mt. Takao, 13.IV.1923, T. Esaki leg. (USNM). 107, 1으, Kanagawa, Yokohama-shi, Asahi-ku, Tsurugamine, Katabira-gawa-Shinsui-ryokudô, Alt. 29 m, 23.IV.2016, T. Mishima leg. 19, Mie, Matsuzaka-shi, Okawachichô, Alt. 73 m, 15.IV.2014. 10', Omi (Shiga), Mt. Ryozen, 30.IX.1958, T. Mishima leg. (USNM). $10^{7}$, Nara, Kamikitayamamura, Nishihara, Namego-dani River, Alt. 800 m, 9.VIII.2015. 2o, Yamaguchi, Iwakuni-shi, Nishiki-machi-Ôno, Mt. Shôjôyama, Alt. $330 \mathrm{~m}, 2 . \mathrm{V} .2016 .10^{7}$, same data as previous except 24.VII.2016. [Shikoku] 1+, Kagawa, Mann-ch, Katsuura, Myojingawa River, Alt. 480 m, 21.IV.2015. 10', (Ehime), Sugitate, 1.XI.1951, T. Ishihara leg. (USNM). 10 $0^{7}$, (Ehime), Matsuyama, 
Dogo, 19.IV.1952, T. Yano leg. (USNM). 1ㅇ, Tokushima, Miyoshi-shi, Higashiiya-Ochiai, near Matsuogawa Dam, Alt. $900 \mathrm{~m}$, 15.V.2015. $20^{\top} \mathrm{O}^{\top}$, same data as previous except 30.IV.2016. 10' Tokushima, Higashimiyoshi-chô, Nishishô, Kamodani-gawa River, Alt. 170 m, 21.IV.2014. 1우, Tokushima, Yoshinogawa-shi, Misato, 16.V.2015. [Kyushu] 10 10,1, Saga, Saga-shi, Fuji-machi-Seiya, Kase-gawa River near Hokuzan Dam, Alt. 320 m, 10.X.2015. $30^{7} 0^{7}, 2$, 2 , same data as previous except 26.X.2015. 1옹 same data as previous except 3.XI.2015. $10^{7}$, same data as previous except 11.XI.2015. 10, same data as previous except 20.XI.2015. [Nansei Islands] Yakushima Is. $60^{7} \sigma^{7}, 19$, unnamed stream near Kigensugi, Alt. 1270 m, 26.IV.2018. Amamiôshima Is.: 10 $0^{7}$, Amami-shi, Naze-OazaDaikuma, seaside, north of Daikuma fishing port, Alt. $2 \mathrm{~m}$, 4.IV.2019. 10 $10^{2}$ 1, Yamato-son, Yuwangama, Alt. $250 \mathrm{~m}$, 31.III.2019. Okinawa Is.: 1엉, Kunigami-son, Yona, Mt. Fuenchijidake, Alt. 300 m, 12.III.2016. Iriomote Is.: 1엉, Iriomote, near Urauchi-gawa River, Alt. $50 \mathrm{~m}$, 14.III.2016. $20^{7} \mathrm{O}^{7}$, Iriomote, Mt. Ûshoku-mori, Alt. 50 m, 14.III.2016. 10², Komi, Mairagawa River, Alt. 20 m, 15.III.2016.

Distribution. Japan (Hokkaido, Honshu, Shikoku, Kyushu, and Nansei Islands) (Fig. 65) and Russian Far East.

Geranomyiafeuerborni(Alexander, 1931)

Figs 18, 31, 43, 55, 66.

Limonia (Geranomyia) feuerborni Alexander, 1931: 163. Type locality: Indonesia, Java, Mt. Lawu, Sarangan.

Geranomyia feuerborni: Kato [2020: 24].

Diagnosis. Dark brownish species with slightly patterned wing. Prescutum usually with two indistinctly dark stripes. Wing with stigma dark brown, bearing three weakly dark tiny spots on costal region (Fig. 18). Legs mainly brown to dark brown, yellowish on segments proximal to bases of femora. Halter dark brown, stem yellowish at base. Abdomen dark brown on tergites. Male terminalia without clasper of gonostylus. Rostral lobe bifid, forming U-shaped notch between branches (Fig. 43).

Distribution. Japan (Nansei Islands) (Fig. 66) and Indonesia.

Geranomyia gifuensis Alexander, 1921

Figs 19, 32, 44, 56, 66.

Geranomyia (Geranomyia) gifuensis Alexander, 1921: 115. Type locality: Japan, Honshu, Gifu.

Limonia (Geranomyia) gifuensis: Alexander [1954: 286].

Geranomyia gifuensis: Nakamura [2014: 46]; Kato and Suzuki [2017: 15].

Diagnosis. Greyish species with slightly patterned wing. Prescutum usually with two dark stripes. Pleuron dusky yellow to dark ocherous. Wing with stigma dark brown, four weakly dark tiny spots on costal region (Fig. 19). Legs mainly dusky yellow to brown, paler on segments proximal to bases of femora. Halter yellowish, knob infuscated. Abdomen dark greyish brown on tergites. Male terminalia with rostral lobe bearing two short spines (Fig. 44). Paramere with lateral part produced posteriorly beyond base of mesal-apical lobe, mesalapical lobe smooth on dorsal margin (Fig. 56).

Material. Paratype: Japan: [Honshu] 19, Gifu, 10.X.1920, K. Takeuchi leg. (USNM). Non-types: Japan: [Honshu] 19, Aomori, Azigasawa-machi, Midstream of Akaishi-gawa River, Alt. 125 m, 4.VII.2014. 19, Aomori, Azigasawa-machi, seaside, 22.IX.2013. 19, Aomori, Nakadomari-machi, Kodomari, river,
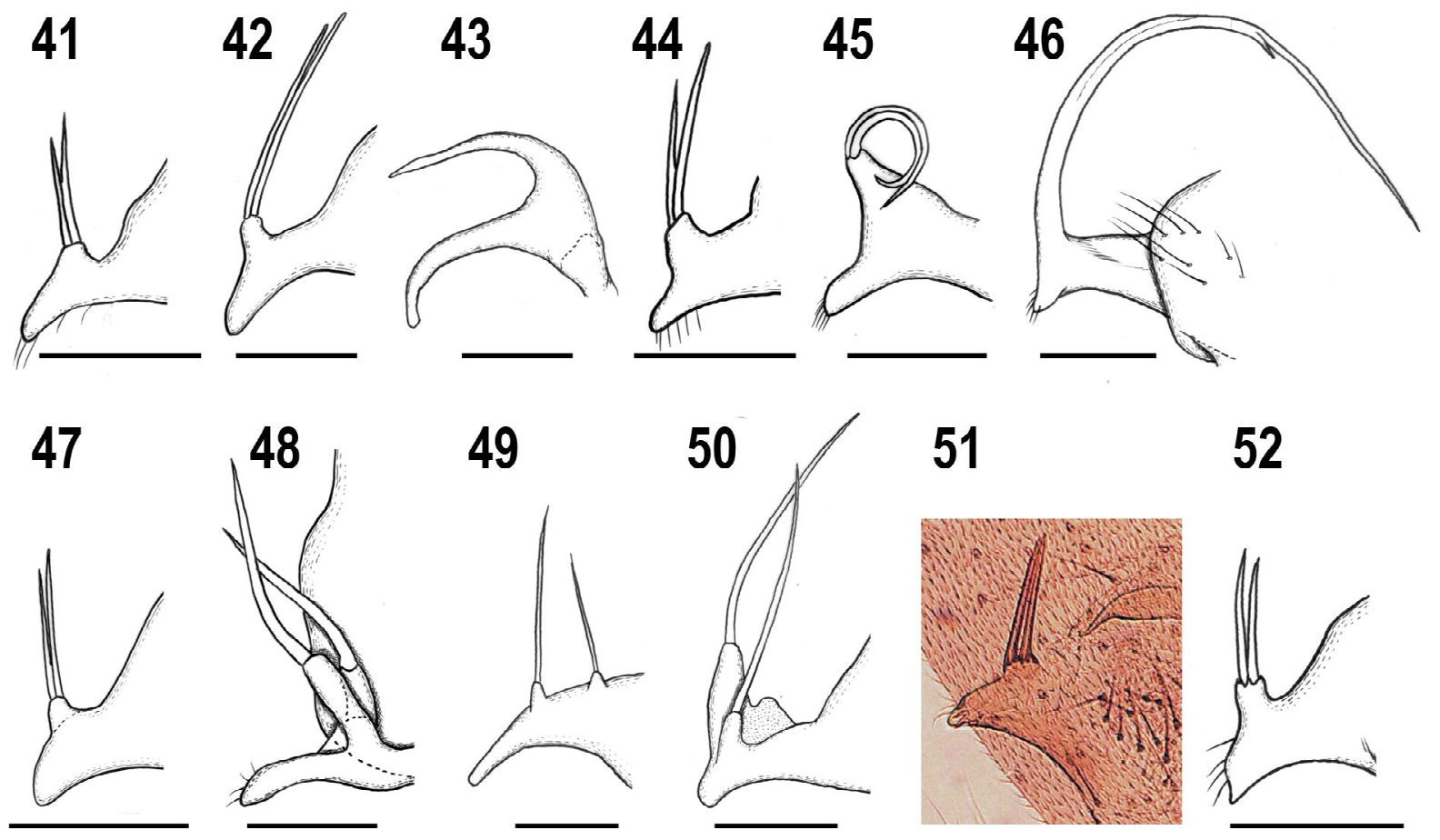
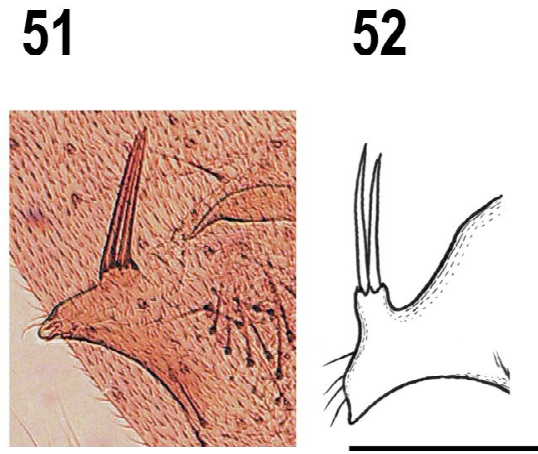

Figs 41-52. Rostral lobes and spines of Japanese Geranomyia spp., dorsal or outer surface (Figs 45-47, 49-52), ventral or inner surface (Figs 41-44, 48): 41 - G. argentifera; 42 - G. avocetta; 43 - G. feuerborni; 44 - G. gifuensis; 45 - G. gravelyana; 46 - G. multipuncta; $47-$ G. pictorum; $48-$ G. radialis; $49-$ G. sakagucbii; $50-$ G. semjina; $51-$ G. snyderi; $52-$ G. sparsiguttata. Scale bars: $0.1 \mathrm{~mm}$.

Рис. 41-52. Вершинные доли и спикулы (дорсально, снаружи рис. 45-47, 49-52, вентрально, изнутри рис. 41-44, 48) японских видов рода Geranomyia spp: 41 - G. argentifera; 42 - G. avocetta; 43 - G. feuerborni; 44 - G. gifuensis; 45 G. gravelyana; 46 - G. multipuncta; 47 - G. pictorum; 48 - G. radialis; 49 - G. sakaguchii; 50 - G. semjina; 51 - G. snyderi; 52 - G. sparsiguttata. Масшта6: 0,1 мм. 

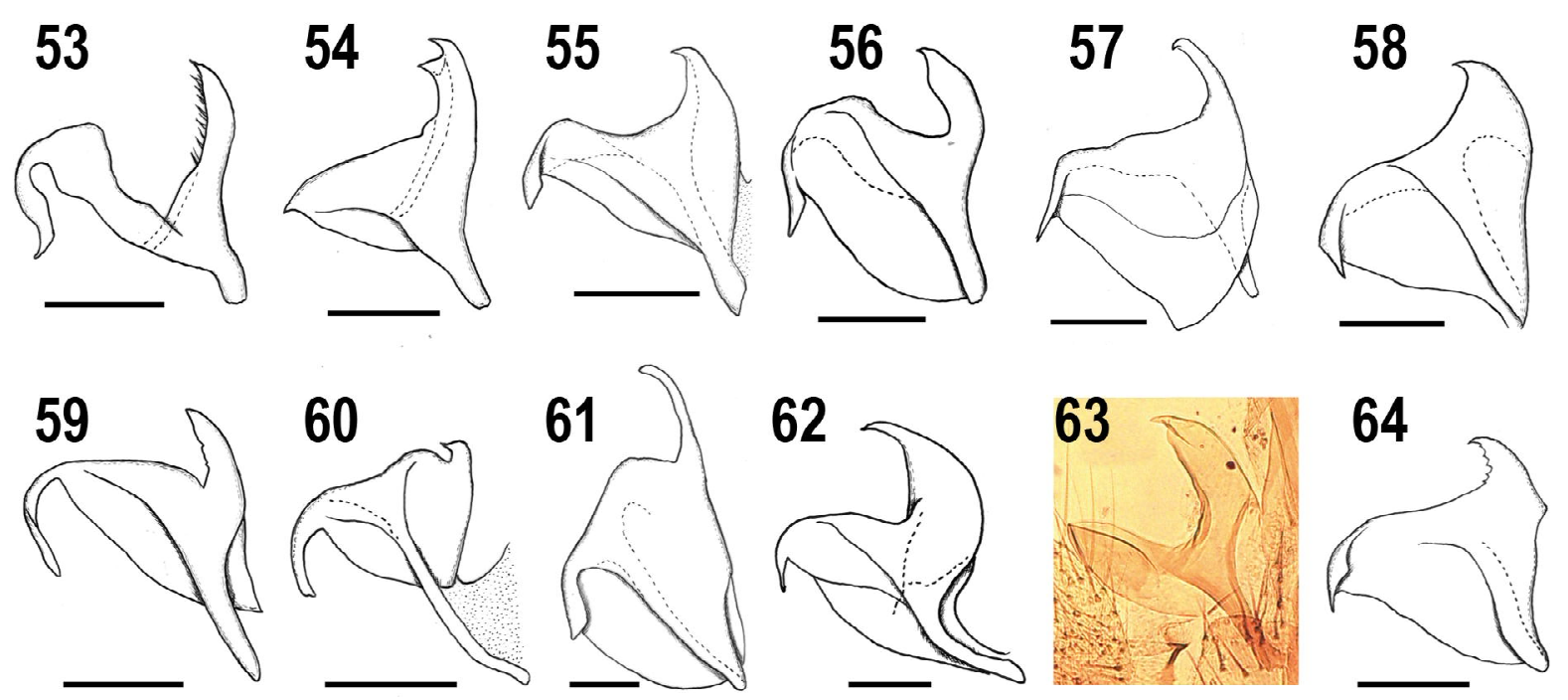

Figs 53-64. Parameres of Japanese Geranomyia spp., ventral or outer surface (Figs 53-62, 64), dorsal or inner surface (Fig. 63): 53 - G. argentifera; 54 - G. avocetta; 55 - G. feuerborni; 56 - G. gifuensis; 57 - G. gravelyana; 58 - G. multipuncta; 59 G. pictorum; 60 - G. radialis; 61 - G. sakaguchii; 62 - G. semjina; 63 - G. snyderi; 64 - G. sparsiguttata. Scale bars: $0.1 \mathrm{~mm}$.

Рис. 53-64. Парамеры (вентрально, снаружи рис. 53-62, 64, дорсально, изнутри рис. 63) японских видов рода Geranomyia spp: 53 - G. argentifera; 54 - G. avocetta; 55 - G. feuerborni; 56 - G. gifuensis; 57 - G. gravelyana; $58-$ G. multipuncta; 59 - G. pictorum; 60 - G. radialis; 61 - G. sakaguchii; 62 - G. semjina; 63 - G. snyderi; 64 - G. sparsiguttata. Масштаб: 0,1 мм.

south of Nanatsudaki-sawa River, Alt. 10 m, 28.VII.2013. 19, Aomori, Nishimeya-mura, Kawaratai, Ôkawa-rindô Path, Alt. 300 m, 4.X.2014. 10 , Aomori, Nishimeya-mura, Kawaratai, The Shirakami Natural Science Park, Hirosaki Univ., Alt. 255 m, 25.X.2014 (LT). 19, Aomori, Hirosaki-shi, Sôma-rindô Path, Alt. 390 m, 28.VI.2013. 1, Aomori, Hachinohe-shi, Okubo, Kanafukizasa Cold Spa, Alt. 85 m, 4.VIII.2013. 10", Akita, Kazuno-shi, Hachimantai, near Hebisawa-numa Pond, Alt. 790 m, 5.VIII.2015. 209, Iwate, Hachimantai-shi, Hosono, Appi Highland, Alt. 520 m, 3.VIII.2013. 10', Echigo (Niigata), Kurokawa, 21.IX.1954, K. Baba leg. (USNM). 10², 1으, Gifu, Nakatsugawa-shi, Kashimo, Nishimata-dani Valley, Alt. 800 m, 7.VIII.2015. 10', Aichi, Seto-shi, Iwaya-chô, near Iwayadô Park, Alt. 300 m, 17.VII.2016. 1우, Ósaka, Mt. Minomo, 4.V.1921, R. Takahashi leg. (USNM). 1우, Okayama, Maniwashi, Hiruzen-Shimotokuyama, Alt. 780 m, 11.VIII.2015. 10", Yamaguchi, Iwakuni-shi, Nishiki-machi-Ôno, Mt. Shôjô-yama, Alt. $330 \mathrm{~m}$, 11.VIII.2015. [Shikoku] 10 Matsuyama, Dogo, 19.IV.1952, Yano leg. (USNM). 19, Iyo (Ehime), Omogo Valley, 9.XI.1953, Mohri leg. (USNM). 10 Tokushima, Higashimiyoshi-chô, Nishishô, Kamodani-gawa River, Alt. 170 m, 21.IV.2014. [Kyushu] 1+, Fukuoka, Fukuokashi, Sawara-ku, Itaya, Mt. Sefuri-san, Alt. 970 m, 2.V.2015. $20^{7} 0^{7}$, same data as previous except 27.VIII.2016 (LT). $30^{7} 0^{7}$, 29ᄋ, Fukuoka, Itoshima-shi, Shimasakurai, Mt. Tenga-dake, Alt. $110 \mathrm{~m}, 29 . \mathrm{V} .2015$. 19, same data as previous except 21.VI.2015. 19, same data as previous except 2.VII.2015. 10" Fukuoka, Miyako-machi, Saigawa-Hobashira, Notoge Pass, Alt. 740 m, 21.IX.2015. 19, Saga, Saga-shi, Fuji-machi-Seiya, Kase-gawa River near Hokuzan Dam, Alt. 320 m, 12.IV.2015.

Distribution. Japan (Honshu, Shikoku, and Kyushu), (Fig. 66), North Korea, South Korea, and Russian Far East.

Geranomyia gravelyana(Alexander, 1942)

Figs 20, 33, 45, 57, 67.

Limonia (Geranomyia) gravelyana Alexander, 1942: 58. Type locality: India, W. Bengal, Darjiling District, Eastern Himalayas.

Geranomyia gravelyana: Kato [2020: 25].
Diagnosis. Greyish species with unpatterned wing except stigma (Fig. 20). Prescutum with four broad dark stripes. Pleuron dusky yellow to dark ocherous. Legs mainly brown to dark brown, paler on segments proximal to bases of femora, fore femur often yellowish entirely. Halter dark brown. Abdomen dark brown on tergites. Male terminalia with rostral lobe bearing two short curled spines (Fig. 45).

Material. Non-types: Japan: [Nansei Islands] Amamiôshima Is.: $2 \sigma^{7} \sigma^{7}, 1$, Yamato-son, Yuwangama, Alt. 250 m, 31.III.2019. Tokunoshima Is.: $20^{7} \sigma^{7}$, Tokunoshima-cho, Todoroki, near Mt. Sasontsuji-dake, Alt. 200 m, 2.IV.2019.

Distribution. Japan (Nansei Islands) (Fig. 67) and India.

Geranomyia hakoneana (Alexander, 1955) Figs 21, 69.

Limonia (Geranomyia) hakoneana Alexander, 1955: 371. Type locality: Japan, Honshu, Kanagawa, Hakone-machi, Miyanoshita.

Geranomyia hakoneana: Nakamura [2014: 46]; Kato and Suzuki [2017: 15].

Diagnosis. Greyish brown species with sparsely patterned wing (Fig. 21). Prescutum with three dark stripes. Pleuron weakly yellowish ventrally. Wing with seven small dark spots on costal region, only stigma large, cord and outer end of cell d seamed with dark brown (Fig. 21). Legs mainly brownish yellow, paler on coxae and trochanters. Halter pale yellow, knob weakly dark at tip. Abdomen dark brown on tergites. Male terminalia with rostral lobe bearing two short spines.

Material. Holotype: $\sigma^{7}$, Japan, Honshu, Hakone, Miyanoshita, 31.VIII.1950, collector not given (USNM).

Distribution. Japan (Honshu) (Fig. 69).

Remarks. Alexander [1955] mentioned that the rostral lobe of the holotype had two straight rostral spines of moderate length as in G. gifuensis but the male terminalia was lost before mounting on a slide. 


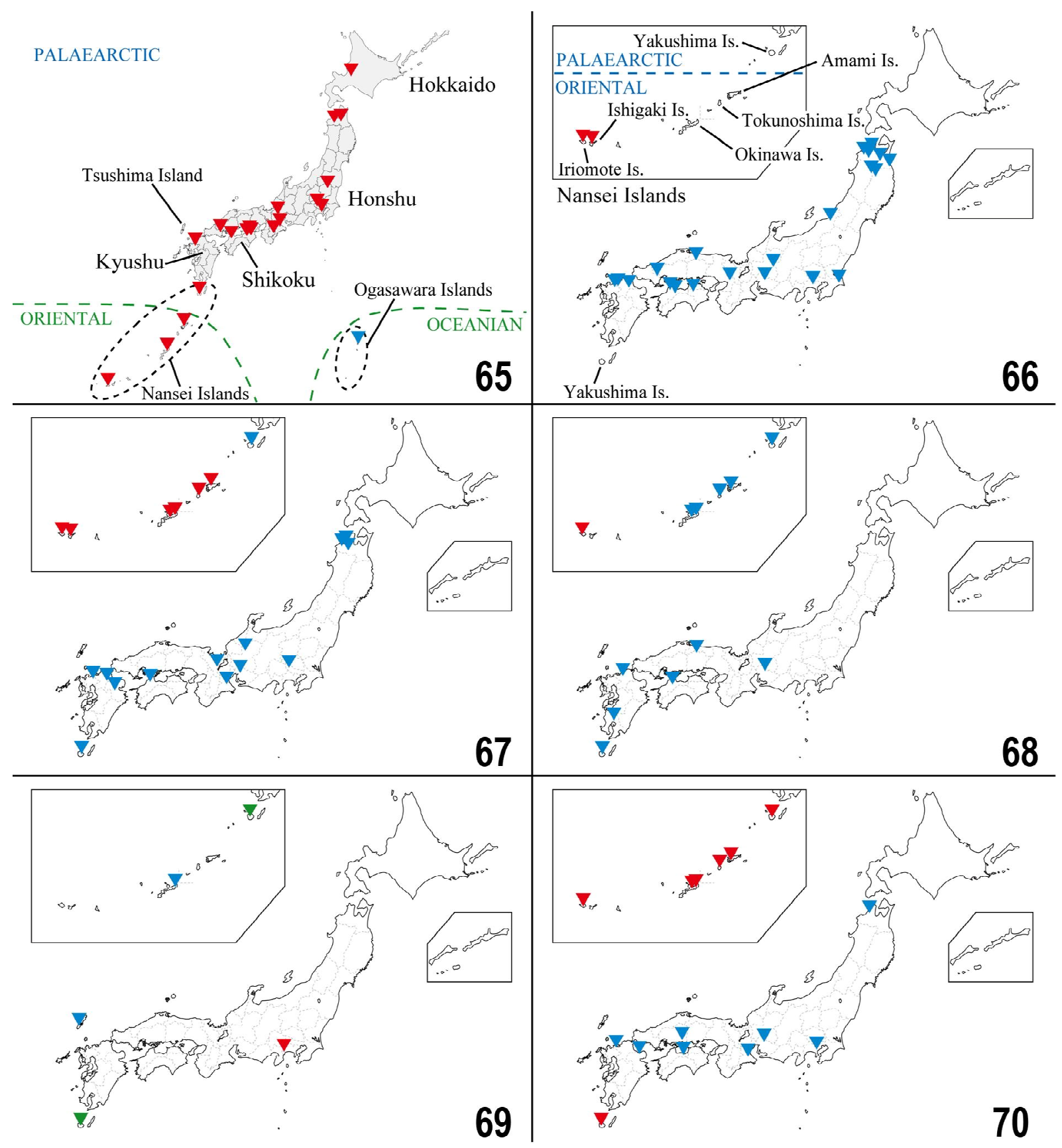

Figs 65-70. Distribution maps of Japanese Geranomyia spp. (data in the previous papers are included): 65 - G. avocetta (red), G. snyderi (blue); 66 - G. argentifera and G. feuerborni (red), G. gifuensis (blue); 67 - G. gravelyana (red), G. multipuncta (blue); 68 - G. iriomotensis sp. n. (red), G. paucipuncta sp. n. (blue); $69-$ G. hakoneana (red), G. sakaguchii (blue), G. pictorum and G. sparsiguttata (green); 70 - G. radialis (red), G. semjina (blue).

Рис. 65-70. Распространение японских видов рода Geranomyia spp. (с учётом данных предыдущих публикаций): $65-$ G. avocetta (красный), G. snyderi (синий); 66 - G. argentifera and G. feuerborni (красный), G. gifuensis (синий); $67-$ G. gravelyana (красный), G. multipuncta (синий); 68 - G. iriomotensis sp.n. (красный), G. paucipuncta sp.n. (синий); 69 G. bakoneana (красный), G. sakaguchii (синий), G. pictorum и G. sparsiguttata (зелёный); 70 - G. radialis (красный), G. semjina (синий). 
Geranomyia multipuncta Alexander, 1922

Figs 22, 34, 46, 58, 67.

Geranomyia multipuncta Alexander, 1922: 177. Type locality: Japan, Honshu, Gifu.

Limonia (Geranomyia) multipuncta: Alexander [1954: 287].

Geranomyia multipuncta: Nakamura [2014: 46].

Diagnosis. Brownish grey to grey species with heavily patterned wing. Prescutum with three dark stripes. Pleuron sometimes yellowish. Wing with seven large dark spots on costal region, cord and outer end of cell d seamed with dark brown, abundant tiny spots on cells proximal to cord (Fig. 22). Legs mainly yellow to brown, sometimes paler on coxae and trochanters. Halter dusky yellow, knob weakly dark. Abdomen brown to dark brown on tergites. Male terminalia with rostral lobe bearing long arched spine, about $1 / 2$ length of clasper of gonostylus (Fig. 34).

Distribution. Japan (Honshu, Shikoku, Kyushu, and Nansei Islands) (Fig. 67) and South Korea.

Material. Holotype: ${ }^{7}$, Japan, Honshu, Gifu, 1.V.1921, K. Takeuchi leg. (USNM). Non-types: Japan: [Honshu] 10', Aomori, Hirosaki-shi, Ichinowatari-Washinosu, Alt. 205 m, 21.V.2013. 1 , , same data as previous except 19.VII.2013. 1의, same data as previous except 5.IX.2013. 10', Aomori, Hirosaki-shi, Koguriyama, Inekari-sawa River, Alt. 170 m, 16.VII.2013. 10", Aomori, Hirosaki-shi, Bunkyô-chô, Hirosaki Univ., 13.X.2013. $10^{\top}$, Aomori, Fukaura-machi, Mt. Takaniô-yama, Alt. $140 \mathrm{~m}$, 11.V.2014. $4 \sigma^{\top} \sigma^{\top}, 2$, 2 , Aomori, Tsugaru-shi, Kizukuri-Dekijima, Seaside, Alt. $5 \mathrm{~m}, 11 . \mathrm{V} .2014 .10^{7}$, same data as previous except 21.VII.2014. 10', Tokyo, Mt. Mitake, 10.V.1931, B. Oda leg. (USNM). 10', Mino (Gifu), Hirugano, 3.V.1959, Mishima leg. (USNM). 1\%, Aichi, Nagoya-shi, Chikusa-ku, Niza-chô, Nagoya Univ., Higashiyama Campus, 15.VI.2016, T. Mishima leg. (BLKU). 1+, Mie, Matsuzaka-shi, Okawachi-chô, Alt. 73 m, 15.IV.2014. 19, Mie, Matsuzaka-shi, Shôchô, Kushida-gawa River, Alt. 25 m, 14.IV.2014. 10', same data as previous except 15.IV.2014. 10', Kyoto, Kibune, 20.IV.1931, Tokunaga leg. (USNM). [Shikoku] 10', Iyo (Ehime), Dogo, Matsuyama, 10.IV.1952, T. Yano leg. (USNM). [Kyushu] 19, Fukuoka, Soeda-machi, Ochiai, Hikosan-gawa River, Alt. 210 m, 22.IV.2016. 1옹, Saga, Saga-shi, Fuji-machi-Seiya, Kase-gawa River near Hokuzan Dam, Alt. 320 m, 12.IV.2015. 10", same data as previous except 10.X.2015. 10 , 1․ Ôita, Yufu-shi, Yufuin-ch-Kawanishi, Alt. 660 m, 16.IV.2016. [Nansei Islands] Yakushima Is.: 1요 Miyanoura, near Sen-no-Ie, Alt. 20 m, 25.IV.2018

\section{Geranomyia pictorum (Alexander, 1929)}

Figs 23, 35, 47, 59, 69.

Geranomyia pulchripennis Brunetti, 1912: 393. Type locality: India, West Bengal, Darjeeling, Kurseong.

Limonia (Geranomyia) pictorum Alexander, 1929: 247. New name for Geranomyia pulchripennis.

Geranomyia pictorum: Kato [2020: 26].

Diagnosis. Light ocherous to brown species with heavily patterned wing. Prescutum with three dark stripes. Pleuron yellowish ventrally. Wing with seven large dark spots on costal region, cord and outer end of cell d seamed with dark brown, abundant tiny spots on cells proximal to cord (Fig. 23). Legs mainly brownish yellow. Halter dark brown, stem ocherous basally. Abdomen brown on tergites. Male terminalia with rostral lobe bearing two short spine (Fig. 47).

Distribution. Japan (Nansei Islands) (Fig. 69), Taiwan, and India.
Geranomyia radialis (Alexander, 1930)

Figs 24, 36, 48, 60, 70 .

Limonia (Geranomyia) radialis Alexander, 1930a: 62. Type locality: Japan, Nansei Islands, Yaku-shima Is., Kosugidani.

Limonia (Geranomyia) radialis: Alexander [1930b: 508].

Geranomyia radialis: Nakamura [2014: 46].

Diagnosis. Light ocherous to grey species with sparsely patterned wing. Prescutum with three dark stripes. Pleuron paler. Wing with seven dark spots on costal region, cord and outer end of cell d seamed with dark brown (Fig. 24). Legs mainly dusky yellow to dark brown, segments proximal to bases of femora paler. Halter dark brown. Abdomen brown to dark brown on tergites. Male terminalia with rostral lobe bearing two long and stout, weakly curved spines (Fig. 48).

Distribution. Japan (Nansei Islands) (Fig. 70), China, and Taiwan.

Material. Holotype: $\sigma^{7}$, Japan, Nansei Islands, Yakushima, Kosugidani, altitude 2,500 feet, 29.IV.1929, S. Issiki leg. (USNM). Paratype: Japan: [Nansei Islands] 10, same data as holotype. Non-types: Japan: [Nansei Islands] Yakushima Is.: 10", near Shirataniunsui-kyô Valley, Alt. 600 m, 27.IV.2018. Amamiôshima Is.: 1잉 Setouchi-chô, Agina, east of Agina-gawa River, Alt. 300 m, 3.IV.2019. 1옹 Setouchi-chô, Shinokawa, Yakugachi-gawa River, Alt. 130 m, 3.IV.2019. Tokunoshima Is.: $10^{7}, 1$, Tokunoshima-cho, Kametsu, near trailhead of Mt. Inokawa-dake, Alt. 300 m, 1.IV.2019. Okinawa Is.: 10", Kunigami-son, Yona, Mt. Fuenchiji-dake, Alt. 300 m, 12.III.2016. 10', Nago-shi, Genka, Mt. Ubashi-yama, Alt. 100 m, 12.III.2016. Iriomote Is.: $2 \sigma^{\top} \sigma^{\top}, 2 \% 0$, Uehara, Urauchi-gawa River, Alt. 50 m, 14.III.2016. 107, Iriomote, near Urauchi-gawa River, Alt. 30 m, 18.V.2016. 1요 Haeminaka, Alt. 170-230 m, 19.III.2016. $10^{7}$, 290, Komi, between Maira- and Shiira-gawa Rivers, Alt. $70 \mathrm{~m}, 29 . \mathrm{I} .2016$.

\section{Geranomyia sakaguchii Alexander, 1924}

Figs 25, 37, 49, 61, 69.

Geranomyia (Geranomyia) sakaguchii Alexander, 1924: 60. Type locality: Japan, Nansei Islands, Okinawa, Okinawa Is., Naha-shi, Shuri.

Geranomyia sakaguchii: Nakamura [2014: 46].

Diagnosis. Greyish ocherous species with sparsely patterned wing. Vertex with small finger-shaped lobe at anterior end. Flagellomeres with middle segments produced ventrally. Prescutum with three dark stripes. Pleuron with two weakly dark stripes. Wing with four dark small spots on anterior region (Fig. 25). Legs mainly yellow. Halter pale yellow. Abdomen brown to dark brown on tergites. Male terminalia with rostral lobe bearing two spines, well separated from each other at bases (Fig. 49).

Distribution. Japan (Tsushima Island and Nansei Islands) (Fig. 69).

Material. Holotype: $\sigma^{7}$, Japan, Nansei Island, Okinawa, Shuri, altitude 300 feet, 11.III.1923, S. Sakaguchi leg. (USNM). Non-types: Japan: [Tsushima Is.] 10', Komoda, 29.IX.1930, Hori and Fujino leg.

Remarks. This species is very similar to a species from Kuril Islands, G. kunashir Podenas, 2016, but is distinguished from it by the following characters: pleuron brownish gray, with two dark stripes (brownish yellow with blackish spot behind cervical sclerite in G. kunashir); tergites without yellowish spots on anterior three segments (yellowish spots present in G. kunashir). 


\section{Geranomyia semjina Podenas, 2016}

Figs 26, 38, 50, 62, 70.

Geranomyia semjina Podenas, 2016: 556. Type locality: South Korea, Jeollanam-do, Gurye-gun, Toji-myeon, Naeseori, Jirisan National Park, Piagol valley.

Geranomyia semjina: Kato [2020: 27].

Diagnosis. Greyish ocherous to grey species with unpatterned wing except stigma (Fig. 26). Vertex without light grey longitudinal line on posterior part. Prescutum with three dark stripes. Pleuron sometimes paler. Legs mainly yellow. Halter yellowish, knob infuscated. Abdomen brown to dark brown on tergites, narrowly yellowish at caudal margins of most segments. Male terminalia with rostral lobe bearing two long and curved spines (Fig. 50).

Distribution. Japan (Honshu, Shikoku, and Kyushu) (Fig. 70) and South Korea.

\section{Geranomyia snyderi (Alexander, 1972)}

Figs 27, 39, 51, 63, 65.

Limonia (Geranomyia) snyderi Alexander, 1972: 783. Type locality: Japan, Ogasawara Islands, Tokyo, Ani Is., Mt. Sen-zan.

Limonia (Geranomyia) snyderi chichiensis Alexander, 1972: 784 , syn. nov. Type locality: Japan, Ogasawara Islands, Tokyo, Chichi Is., Ô-mura.

Geranomyia snyderi chichiensis Nakamura [2014: 47].

Geranomyia snyderi snyderi Nakamura [2014: 47].

Diagnosis. Dark greyish brown species with sparsely patterned wing. Prescutum ocherous, with three broad dark stripes, often fused with one another. Pleuron yellow to ocherous on dorsal half of katepisternum. Wing with three small dark costal spots and larger stigma (Fig. 27). Legs mainly dusky yellow, segments proximal to bases of femora paler, each tibia with broad, distinctly dark area at tip, more than four times as long as wide. Halter dark brown. Abdomen dark brown on tergites. Male terminalia with rostral lobe bearing two short spines (Fig. 51).

Distribution. Japan (Ogasawara Islands) (Fig. 65).

Material. Holotypes: $\sigma^{7}$, Japan, Ogasawara Islands, Chichi Gr., Ani Is., Sen-zan, Northeast Bay, 28.V.1958, Snyder leg. (holotype of Limonia (Geranomyia) snyderi / USNM); 19, Japan, Ogasawara Islands, Chichi Is., Gr., Omura, «camp beach», 2-25.IV.1958, Snyder leg. (holotype of Limonia (Geranomyia) snyderi chichiensis / USNM). Paratype: Japan: [Ogasawara Islands] 1, Tokyo, Chichi Is., Yatsuse River, Minatoko, 10-22.IV.1958, F. M. Snyder leg. (USNM). Nontypes: Japan: [Ogasawara Islands] Chichi Is.: 2+o, northern tributary of Shigure Dam, Alt. 80 m, 15.V.2018. 4우, ChichijimaKitafukurozawa, Tokoyono-taki Waterfall, Alt. 35 m, 16.V.2018.

Remarks. A subspecies of this species, G. snyderi chichiensis, from Chichi Is., which is situated about $500 \mathrm{~m}$ north of the type locality of the nominotypical subspecies, Ani Is., was separated by body size, length of rostrum, coloration of thorax, and wing pattern. However, these differences are slight and often occur within species of Geranomyia. As a result of examining additional specimens from Chichi Is., the darker coloration on the thorax of the type of Geranomyia snyderi chichiensis was turned out to be due to decay and discoloration. Therefore this subspecies is synonymized with the nominotypical species in this study.

\section{Geranomyia sparsiguttata (Alexander, 1937)} Figs 28, 40, 52, 64, 69.

Limonia (Geranomyia) sparsiguttata Alexander, 1937: 374. Type locality: China, Fujian, Liung-chon-san.

Geranomyia sparsiguttata: Kato [2020: 28].
Diagnosis. Greyish brown species with sparsely patterned wing. Prescutum with three dark stripes. Pleuron dusky yellow on ventral half. Wing with weakly dark, four small costal spots and larger stigma (Fig. 28). Legs mainly brown, segments proximal to bases of femora paler. Halter dark brown. Abdomen dark brown on tergites. Male terminalia with rostral lobe bearing two short spines (Fig. 52). Paramere with posterior end of lateral part at almost same level of base of mesal-apical lobe, mesal-apical lobe serrate on dorsal margin (Fig. 64).

Distribution. Japan (Nansei islands) (Fig. 69) and China.

\section{Key to JAPANESE SPECIES OF GERANOMYIA}

1. Vertex with finger-shaped lobe at anterior end; most ofmale flagellomeres produced ventrally, with short necks at anterior and posterior ends; wing with four small spots (Fig. 25); lobe of gonostylus small, shorter than gonocoxite, clasper of gonostylus almost straight (Fig. 37) G. sakaguchii

- Vertex without finger-shaped lobe; most of male flagellomeres bacilliform, without necks at anterior and posterior ends

2. Anterior vertex at narrowest point more than 2.5 times as wide as scape; prescutum with two slivery spots on lateral margin; wing unpatterned except stigma (Fig. 16); mesalapical lobe of paramere bearing row of setae (Fig. 53) ... G. argentifera

- Anterior vertex at narrowest point at most 1.5 times as wide as scape; prescutum without silvery spots ......... 3

3. Posterior part of vertex without central grayish line in anterodorsal view; wing unpatterned except stigma (Fig. 26); lobe of gonostylus about as long as gonocoxite (Fig. 38); rostral lobe with two long rostral spines from two tubercles (Fig. 50)..... G. semjina

- Posterior part of vertex with grayish middle line in anterodorsal view ... 4

4. Wing posterior margin with more than one spot proximal to tip of $\mathrm{M}_{4}$ (Figs 17, 21-23) ....................................... 5

- Wing posterior margin without spot or with one spot at tip of $A_{1}$ (Figs 2, 9-10, 18-20, 24, 27, 28) ................. 8

5. Cell bm without spots (Figs 17, 21) ........................... 6

- Cell bm with group of small dark spots (Figs 22-23) ..... 7

6. Spots on wing except stigma roundish, much smaller than 1/4 of stigma (Fig. 21) G. hakoneana

- Spots on wing except stigma squarish, some spots about half as large as stigma (Fig. 17) .................... G. avocetta

7. Spot at tip of Sc at least as large as one at origin of Rs, extending to Rs (Fig. 23); male terminalia with two short and straight rostral spines (Fig. 47) ... G. pictorum

- Spot at tip of Sc smaller than one at origin of Rs, not reaching at Rs (Fig. 22); male terminalia with one long and curved rostral spine (Fig. 46) G. multipuncta

8. Tibia with distinctly dark area at tip, more than four times as long as wide; paramere with mesal-apical lobe large, about half length of paramere (Fig. 63) ......... G. snyderi

- Tibia without dark apical area or with much shorter weakly dark area at tip; paramere with mesal-apical lobe at most $1 / 3$ length of paramere (Figs 7, 15, 55-57, 60, 64) ...... 9

9. Wing (Fig. 24) with seven costal spots; costal spots at origin of Rs and level of tip of $A_{1}$ rectangular, both almost reaching at $\mathrm{M}$; crossvein $\mathrm{m}$-cu usually situated more than 
halflength of itself proximal to inner end of cell d; two long rostral spines present from two tubercles (Fig. 48) ........

G. radialis

- Wing usually with less than seven costal spots; if seven costal spots present, spot at origin of Rs roundish, not apparently reaching at $\mathrm{M}$; crossvein $\mathrm{m}$-cu usually within half length of itself from inner end of cell d; rostral spine(s) not as above

.. 10

10. Clasper of gonostylus absent (Fig. 31); one stout rostral spine present, directed dorsally (Fig. 43)

G. feuerborni

- Clasper of gonostylus present, slender sickle-shaped (Figs $3,11,32-33,40)$

11. One rostral spine present (Figs 5, 13) ....................... 12

- Two rostral spines present (Figs 44-45, 52) ............. 13

12. Rostral spine about as long as clasper of gonostylus (Figs. 3,5 ); paramere gradually narrowed to tip (Fig. 7)

G. iriomotensis sp.n.

- Rostral spine about twice as long as clasper of gonostylus (Figs. 11,13); paramere strongly narrowed on mesal-apical lobe (Fig. 15) G. paucipuncta sp.n.

13. Rostral spines curled (Fig. 45) ............... G. gravelyana

- Rostral spines straight (Figs 44, 52) 14

14. Lobe of gonostylus about 3.5 times as long as gonocoxite (Fig. 40); lateral part of paramere not produced posteriorly beyond base of mesal-apical lobe, dorsal margin of mesalapical lobe serrate (Fig. 64). G. sparsiguttata

- Lobe of gonostylus about twice as long as gonocoxite (Fig. 32); lateral part of paramere produced posteriorly beyond base of mesal-apical lobe, dorsal margin of mesal-apical lobe smooth (Fig. 56) G. gifuensis

\section{Acknowledgements}

I am indebted to Dr. Jon Gelhaus (the Academy of Natural Science of Drexel University, Philadelphia, USA) for helping me examine specimens in USNM and to Dr. Floyd Shockley and Dr. Torsten Dikow (USNM) for allowing me to examine their specimens. I thank Dr. Tatsuya Mishima and Dr. Takuji Tachi (Biosystematics Laboratory, Faculty of Social and Cultural Studies, Kyushu University, Japan) for providing valuable specimens and comments on the early draft of this paper. I was supported during the stay in USA for examining specimens by the Japan Student Services Organization (JASSO) Japan Public-Private Partnership Student Study Abroad Program (Young Ambassador Program).

\section{References}

Alexander C.P. 1913. Report on a collection of Japanese craneflies (Tipulidae), with a key to the species of Ptychoptera [part] // Canadian Entomologist. Vol.45. P.197-210.

Alexander C.P. 1921. Undescribed species of Japanese craneflies (Tipulidae, Diptera). Part II // Annals of the Entomological Society of America. Vol.14. P.111-134.

Alexander C.P. 1922. New species of Japanese crane-flies. Part III. (Diptera, Tipulidae) // Insecutor Inscitiae Menstruus. Vol.10. P.177-188.

Alexander C.P. 1924. Undescribed species of Japanese craneflies. Part IV // Annals of the Entomological Society of America. Vol.17. P.59-74.
Alexander C.P. 1929. New or little-known Tipulidae from the Philippines (Diptera). V // Philippine Journal of Science. Vol.40. P.239-273.

Alexander C.P. 1930a. New or little-known Tipulidae from eastern Asia (Diptera). VI // Philippine Journal of Science. Vol.42. P.59-83.

Alexander C.P. 1930b. New or little-known Tipulidae from eastern Asia (Diptera). VII // Philippine Journal of Science. Vol.42. P.507-535

Alexander C.P. 1931. Deutsche Limnologische SundaExpedition. The crane-flies (Tipulidae, Diptera) // Archiv fur Hydrobiologie, Suppl.-Bd. Vol.9 (Tropische Binnengewasser, Band II). P.135-191.

Alexander C.P. 1937. New or little-known Tipulidae from eastern Asia (Diptera). XXXV // Philippine Journal of Science. Vol.63. P.365-404.

Alexander C.P. 1942. The Oriental Tipulidae in the collection of the Indian museum. Part II // Records of the Indian Museum. Vol.44. P.29-72.

Alexander C.P. 1953. Records and descriptions of Japanese Tipulidae (Diptera). Part II. The crane-flies of Shikoku. II // Philippine Journal of Science. Vol.82. P.141-179.

Alexander C.P. 1954. Records and descriptions of Japanese Tipulidae (Diptera). Part III. The crane-flies of Shikoku. III // Philippine Journal of Science. Vol.82. P.263-308.

Alexander C.P. 1955. Undescribed species of Japanese crane-flies (Diptera: Tipulidae). Part VIII // Annals of the Entomological Society of America. Vol.48. P.364374.

Alexander C.P. 1972. Diptera: Tipulidae // Insects of Micronesia. Vol.12. P.733-863.

Brunetti E. 1912. Diptera Nematocera (excluding Chironomidae and Culicidae) // Shipley A.E., Marshall G.A.K. (Eds): Fauna of British India, including Ceylon and Burma. Taylor and Francis. London. P.1-581.

Cumming J.F., Wood D.M. 2017. Adult morphology and terminology // Kirk-Spriggs A.H., Sinclair B.J. (Eds): Manual of Afrotropical Diptera. Vol.1. Introductory chapters and keys to Diptera families. Suricata. Vol.4. South African National Biodiversity Institute. Pretoria. P.89-133.

Gavryushin D.I. 2018. Contribution to the knowledge of limoniid flies (Diptera: Limoniidae) from the Dominican Republic, with the description of a new species of Geranomyia Haliday, 1833 and 12 newly recorded species // Russian Entomological Journal. Vol.27. P.61-66.

Kato D. 2020. New records of Japanese Limoniidae (Diptera: Limoniidae) // Makunagi. Acta Dipterologica. Vol.31. P.1552

Kato D., Suzuki, Y. 2017. A revised list of the crane flies of Kanagawa, Japan, with newly recorded species (Diptera, Tipuloidea) // Makunagi. Acta Dipterologica. Vol.28. P.724

Meijere J.C.H. de 1911. Studien uber Sudostasiatische Dipteren, V. Ostindische Tipulidae // Tijdschrift voor Entomologie. Vol.54. P.21-79.

Nakamura T. 2002. Diptera of the Nasu Imperial Villa, Tochigi, Japan // Tochigi Prefectural Museum (Eds). Flora and Fauna of the Nasu Imperial Villa. Tochigi Prefectural Museum. Utsunomiya. P.167-178. [In Japanese with English title].

Nakamura T. 2014. Family Limoniidae // Nakamura T., Saigusa T., Suwa M. (Eds). Catalogue of the Insects of Japan Volume 8 Diptera, Part 1 Nematocera - Brachycera Aschiza. Touka Shobo. Fukuoka. P.9-53.

Oosterbroek P. 2020. Catalogue of the Craneflies of the World (Diptera, Tipuloidea: Pediciidae, Limoniidae, Cylindrotomidae, Tipulidae). Version 14 Nov. 2020. Online version at https://ccw.naturalis.nl/index.php. 
Podenas S. 2016. New Geranomyia crane flies (Diptera: Limoniidae) from Korea and Kunashir Island // Zootaxa. Vol.4121. P.555-565.

Qian X., Zhang X. 2020. Two new Geranomyia Haliday (Diptera, Limoniidae) crane flies from Mount Jiulong in China, with an updated key to Chinese species // Zookeys. Vol.953 P. $105-118$.
Ribeiro G.C. 2006. Homology of the gonostylus in crane flies, with emphasis on the families Tipulidae and Limoniidae (Diptera, Tipulomorpha) // Zootaxa. Vol.1110. P.47-57.

Zhang X., Zhang Z., Yang D. 2016. Five new species of Geranomyia Haliday, 1833 (Diptera, Limoniidae) from China // Zootaxa. Vol.4154. P.139-154.

Поступила в редакцию 15.12.2020 